\title{
ARTICLE \\ Serum biomarkers combined with ultrasonography for early diagnosis of non-alcoholic fatty liver disease confirmed by magnetic resonance spectroscopy
}

\author{
Ling-ling Qian ${ }^{1,2}$, Liang Wu ${ }^{1}$, Lei Zhang ${ }^{1,2}$, Jing Zhang ${ }^{1,2}$, Jia Zhou ${ }^{3}$, Yue-hua $\mathrm{Li}^{3}$, Qi-chen Fang ${ }^{1}$, Hua-ting $\mathrm{Li}^{1}$ and Wei-ping Jia ${ }^{1}$
}

\begin{abstract}
Magnetic resonance spectroscopy (MRS) is notably accurate for even minimal degree of hepatic steatosis in non-alcoholic fatty liver disease (NAFLD). But routine use of MRS is limited by its cost and availability. In this study, we developed a diagnostic model combining ultrasonography with biomarkers to identify mild NAFLD, with MRS as the reference standard. A total of 422 eligible subjects were enrolled. The serum levels of fibroblast growth factor 21 (FGF21), cytokeratin 18 M65ED, proteinase 3, neutrophil elastase, alpha- 1 antitrypsin, and neutrophil elastase/alpha-1 antitrypsin were measured using ELISA assays. We found that among the six biomarkers, only serum FGF21 was independently associated with intrahepatic triglyceride content (IHTC, standardized $\beta=$ $0.185, P<0.001)$ and was an independent risk factor for mild NAFLD. Thus, we established a Mild NAFLD Model based on FGF21, alanine transaminase, triglycerides, and body mass index. The area under the receiver-operating characteristic curve of the Mild NAFLD Model was 0.853 (95\% confidence interval: 0.816-0.886). Furthermore, a two-step approach combining ultrasonography with the Mild NAFLD Model displayed a better sensitivity for diagnosing mild NAFLD compared with each method alone, with a sensitivity of $97.32 \%$ and a negative predictive value of $85.48 \%$. This two-step approach combining ultrasonography and the Mild NAFLD Model derived from serum FGF21 improves the diagnosis of mild NAFLD and can be applied to the early diagnosis of NAFLD in clinical practice.
\end{abstract}

Keywords: fatty liver; non-alcoholic fatty liver disease; biomarkers; diagnostic model; fibroblast growth factor 21; ultrasonography; magnetic resonance spectroscopy

Acta Pharmacologica Sinica (2020) 41:554-560; https://doi.org/10.1038/s41401-019-0321-x

\section{INTRODUCTION}

In 2018, 25\% of the world's population was estimated to have non-alcoholic fatty liver disease (NAFLD); the prevalence of NAFLD in Asia ranges from $15 \%$ to $40 \%$ and is accompanied by a serious public and economic burden [1]. Hepatic steatosis is generally graded as minimal $(<5 \%)$, mild $(5 \%-33 \%)$, moderate $(33 \%-66 \%)$, and severe $(\geq 66 \%)$ steatosis [2], with the mild grade accounting for the highest proportion of NAFLD patients in population-based studies [3, 4].

Because of the high prevalence and asymptomatic presentation of NAFLD, the early diagnosis of NAFLD is clinically important. First, as it is not always benign, simple steatosis can even evolve to non-alcoholic steatohepatitis (NASH) or progress to fibrosis, cirrhosis, and hepatocellular carcinoma $[5,6]$. Second, the severity of NAFLD increases the risk of impaired fasting glucose, diabetes, microvascular diabetic complications, and cardiovascular diseases $[7,8]$. Third, even a low grade of NAFLD can affect adherence to interventional studies among NAFLD patients [9]. Moreover, this condition is often overlooked in healthy controls selected for clinical trials because of its asymptomatic presentation leading to misclassifications and decreased study validity [10]. Hence, much attention should be paid to the early detection of NAFLD.

Liver biopsy, the gold standard for identifying NAFLD, is invasive; thus, the uses of noninvasive alternatives, such as imaging studies, are necessary for assessing NAFLD. Ultrasonography, the most commonly used noninvasive method, is accurate and reliable for detecting moderate-to-severe fatty liver, but it is known to underestimate NAFLD with intrahepatic fat $<30 \%$ [11]. Computed tomography $(\mathrm{CT})$ is associated with radiation exposure, and it is also not accurate in assessing mild NAFLD [12]. Magnetic resonance spectroscopy (MRS) is highly accurate for even minimal amounts of steatosis, but its widespread application is hampered by time-consuming acquisitions and complex postprocessing procedures [13, 14].

Some biomarkers also hold promise in diagnosing NAFLD, but their roles in diagnosing mild NAFLD defined by MRS have rarely been explored or compared. Fibroblast growth factor 21 (FGF21) is a hepatokine that is often increased in obesity, metabolic syndrome, type 2 diabetes, and NAFLD $[15,16]$. Serum FGF21 was positively correlated with hepatic fat content in subjects with

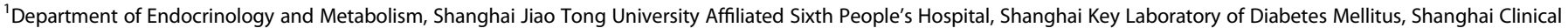

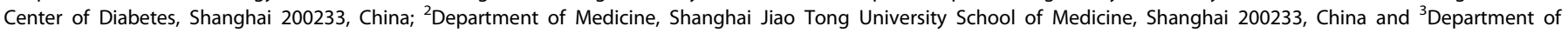
Radiology, Shanghai Jiao Tong University Affiliated Sixth People's Hospital, Shanghai 200233, China

Correspondence: Hua-ting Li (huarting99@sjtu.edu.cn) or Wei-ping Jia (wpjia@sjtu.edu.cn)

These authors contributed equally: Ling-ling Qian, Liang Wu

Received: 6 June 2019 Accepted: 17 October 2019

Published online: 27 November 2019 
mild/moderate NAFLD [17]. Moreover, it has been reported that the neutrophil serine proteases involved in the induction of inflammation and apoptosis play crucial roles in the pathogenesis of obesity-induced NAFLD [18-22]. While several studies have evaluated serum FGF21, apoptosis markers, such as cytokeratin 18 M65ED (CK18 M65ED) and neutrophil serine proteases (proteinase 3 [PR3], neutrophil elastase [NE]), and their inhibitor (alpha-1 antitrypsin $[A 1 A T])$ in relation to the development of NAFLD, no studies have focused on the role of these biomarkers in diagnosing mild NAFLD.

This study aimed to investigate the relationship between novel serum hepatic biomarkers and liver steatosis grades as measured by MRS and assess the diagnostic performance of NAFLD, including novel hepatic markers, in combination with ultrasonography for NAFLD.

\section{MATERIALS AND METHODS}

Study participants

The data of the 531 subjects who underwent ultrasonography and MRS from the local community in Shanghai between October 2017 and October 2018 were reviewed retrospectively to identify Chinese subjects aged 18-70 years with an intrahepatic triglyceride content (IHTC) $<33 \%$. In this project, overweight or obese subjects, as defined by body mass index (BMI) $\geq 24 \mathrm{~kg} / \mathrm{m}^{2}$ or waist circumference (WC) $\geq 85 \mathrm{~cm}$ in men and $\geq 80 \mathrm{~cm}$ in women, were chosen as the high-risk subgroup from the local community. Subjects did not have any known chronic or acute liver diseases, such as autoimmune disease, celiac disease, Wilson's disease, A1AT deficiency, hepatic malignancies, hepatobiliary infections, or biliary tract disease. All the subjects were asked to undergo the ultrasonography examination, and then were invited to quantify their IHTC with an MRS examination. Of 470 eligible subjects, 48 were excluded (12 because they had excessive alcohol consumption [men $>140 \mathrm{~g} /$ week, women $>70 \mathrm{~g} /$ week], 16 with hepatitis infection, 17 with known hyperthyroidism or hypothyroidism, and 3 without serum samples). The final study population consisted of 422 subjects. Of these, 336 individuals had an IHTC more than $5.56 \%$, and 86 individuals had an IHTC less than $5.56 \%$. All of the participants provided informed consent, and the local ethics committee approved the protocol of the study.

Anthropometric and biochemical measurements

Blood pressure, body weight, height, WC, and biomedical indices were measured according to our previous standardized protocols [16]. BMI (weight $[\mathrm{kg}] /$ height $^{2}\left[\mathrm{~m}^{2}\right]$ ) was also calculated. Blood samples were collected from participants after an overnight fast of at least $10 \mathrm{~h}$ and were used to measure serum fasting plasma glucose (FPG), fasting insulin (FINS), fasting $C$ peptide (FCP), alanine transaminase (ALT), aspartate aminotransferase (AST), gamma-glutamyl transferase (GGT), triglyceride (TG), total cholesterol (TC), high-density cholesterol (HDL-C), and low-density cholesterol (LDL-C). Two-hour plasma glucose was measured following a $75-\mathrm{g}$ oral glucose tolerance test. The homeostasis model assessment of insulin resistance (HOMA-IR) was calculated as follows: $\mathrm{HOMA}-\mathrm{IR}=\mathrm{FPG}(\mathrm{mmol} / \mathrm{L}) \times \mathrm{FINS}(\mathrm{mU} / \mathrm{L}) / 22.5$.

Measurement of FGF21, CK18 M65ED, PR3, NE, and A1AT Concentrations of FGF21, PR3, NE, and A1AT in serum were quantified using an enzyme-linked immunosorbent assay (ELISA) kit (Antibody and Immunoassay Services, the University of Hong Kong). Serum CK18 M65ED concentration was quantified with the M65 EpiDeath ELISA kit (Peviva AB, Bromma, Sweden). The intraassay variations for the measurement of FGF21, PR3, NE, A1AT, and CK18 M65ED were $1.89 \%, 4.25 \%, 8.61 \%, 5.97 \%$, and $0.77 \%$, respectively, and for interassay variations, these values were $4.08 \%, 2.45 \%, 4.86 \%, 2.52 \%$, and $8.23 \%$, respectively.
Ultrasonography and MRS examination

After an overnight fast of at least $10 \mathrm{~h}$, all subjects underwent ultrasonography examination in the morning by two experienced sonographers who were blinded to the clinical data. The sonographers performed abdominal ultrasonographic examinations in all subjects using a Voluson 730 Expert B-mode ultrasound machine (GE Healthcare, 5.0-MHz transducer, Waukesha, WI, USA). In accordance with the standards we used in our previous study, we had followed the guidelines for the assessment and management of NAFLD in the Asia-Pacific region [16]. Diffuse fatty liver can be defined by the presence of at least two of three abnormal findings on abdominal ultrasonography (diffusely increased echogenicity ('bright') in the liver with liver echogenicity greater than kidney or spleen, vascular blurring, and deep attenuation of ultrasound signal). Any disagreement between the two sonographers was resolved by consensus.

Subjects underwent liver MRS by the segmented breath-hold method using the 3.0-T Philips Ingenia medical system (Philips Healthcare, the Netherlands) and the 16-channel body coil. Single breath-hold single-voxel MRS data were acquired in midexpiration using point-resolved single-voxel spectroscopy pulse sequence without water suppression with the following parameters: repetition time, $2000 \mathrm{~ms}$; echo time, $50 \mathrm{~ms}$; flip angle, 90; 2 dynamic scans, dynamic scan time, $12 \mathrm{~s}$; and voxel size, $20 \mathrm{~mm} \times 20 \mathrm{~mm} \times 20 \mathrm{~mm}$. Sagittal, coronal, and axial slices were acquired, and regions of interest in the right posterior lobe were selected by an experienced radiologist, avoiding visible blood vessels and bile ducts. All the raw MR spectrum images were processed by two experienced radiologists independently who were blinded to the clinical histories using the standard Philips curve-fitting package. Automatic processing included phase correction based on the water and 200-ms exponential filter. Manual processing included optimization of the phase and a linear baseline correction, if required. The average IHTC derived from the two analyzers was expressed as a percentage by dividing the integral of the methylene groups in the fatty acid chains of the hepatic TG by the sum of the methylene groups and water. Any disagreement between the two analyzers was resolved by consensus.

\section{Diagnostic criteria for NAFLD, mild NAFLD, and metabolic syndrome}

The diagnostic criteria for NAFLD and metabolic syndrome were in accordance with the standards we followed in our previous study [16]. All subjects were invited to confirm the diagnosis of NAFLD via an accurate quantification by MRS (IHTC $\geq$ 5.56\%) [23]. Mild NAFLD was defined as NAFLD with IHTC less than $33 \%[2]$.

\section{Statistical analysis}

All analyses were performed with SPSS version 25.0 (Chicago, IL, USA). Normally distributed data were expressed as the mean \pm SD. Data that were not normally distributed were log-transformed before analysis and were expressed as medians with interquartile ranges. Student's unpaired $t$ test was used for between-group comparisons. Pearson's chi-square and partial correlation analyses were used to examine the associations between IHTC and various parameters. Multiple stepwise regression analysis was used to examine the association of IHTC with other parameters. Multiple logistic regressions were used to identify independent risk factors for mild NAFLD. The accuracy of the models was evaluated using the area under the curve (AUC) with $95 \% \mathrm{Cl}$. Two-sided $P$ values < 0.05 were considered significant. The sensitivity, specificity, positive predictive value, and negative predictive value were calculated to assess the diagnostic accuracy of different diagnostic models. Chi-square tests were used to compare the difference in diagnostic accuracy. 
Table 1. The general characteristics of the subjects with NAFLD in 422 subjects.

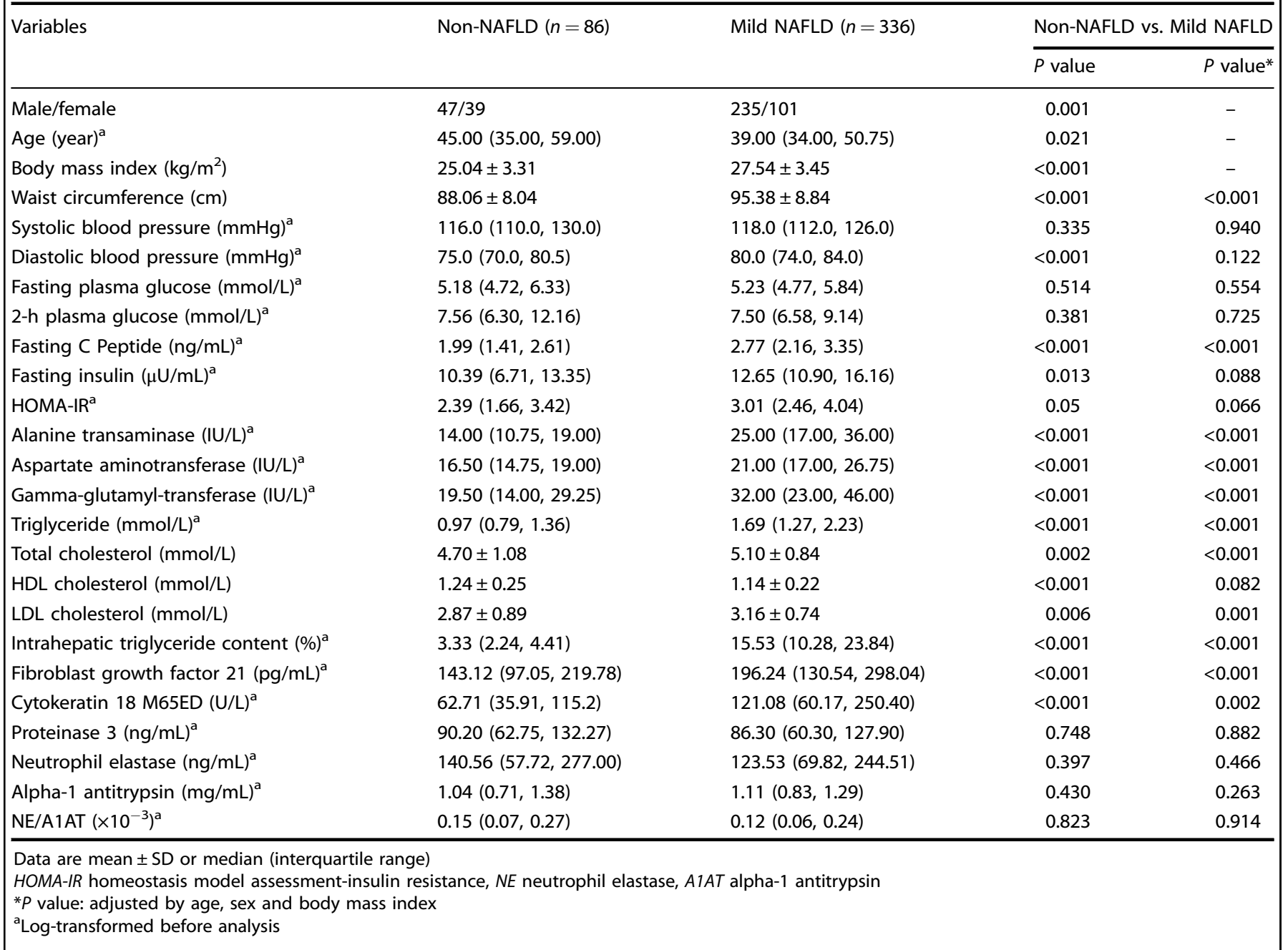

\section{RESULTS}

Characteristics of subjects and serum levels of biomarkers

The clinical characteristics of the 422 subjects included in this study are described in Table 1. Their average age and BMI were 41.00 years and $27.06 \mathrm{~kg} / \mathrm{m}^{2}$, respectively. No significant differences in age were observed between non-NAFLD and mild NAFLD subjects. The male to female ratio and BMI were significantly higher in the mild NAFLD subjects than in the non-NAFLD subjects (both $P<0.05$ ). After adjustment for age, sex, and BMI, subjects with mild NAFLD had a higher WC, FCP, ALT, AST, GGT, TG, TC, LDL-C, and IHTC (all $P<0.01$ ).

Regarding changes in the six biomarkers, both FGF21 and CK18 M65ED levels were significantly higher in patients with mild NAFLD than in the non-NAFLD subjects after adjustment for age, sex, and BMI $(P<0.001$ and $P=0.002$, respectively). Other biomarkers, including PR3, NE, A1AT, and NE/A1AT, did not differ significantly between the two groups. Among the subjects diagnosed with non-NAFLD by ultrasonography, those with MRS-defined NAFLD had significantly higher serum levels of FGF21 than non-NAFLD subjects $(196.24$ [130.54-298.04] pg/mL vs. 143.12 [97.05-219.78] $\mathrm{pg} / \mathrm{mL}, P<0.01)$, with and without adjustment for BMI.

Relationship between biomarkers and IHTC

As IHTC is a quantitative index for the severity of NAFLD, we investigated the relationship between IHTC and the six biomarkers to identify an appropriate biomarker for mild NAFLD. Correlations between IHTC and biomedical parameters are presented in Table 2. After adjustment for age, sex, and BMI, IHTC was positively correlated with WC, FCP, ALT, AST, GGT, TG, TC, highdensity lipoprotein cholesterol (HDL-C), LDL-C, FGF21, and CK18 M65ED (all $P<0.05$ ). Notably, no significant associations were found between IHTC and the other four markers. Multiple stepwise regression analysis involved the above parameters; variables with significant correlations with IHTC included BMI, WC, systolic blood pressure, diastolic blood pressure, FCP, ALT, AST, GGT, TG, HDL-C, FGF21, and CK18 M65ED. FGF21 (standardized $\beta=0.185, P<0.001$ ), FCP, ALT, and TG were found to be independently associated with IHTC. However, CK18 M65ED did not demonstrate a significant association.

FGF21 is an independent risk factor for mild NAFLD diagnosed by MRS

To determine whether FGF21 was an independent risk factor for NAFLD, we performed a multiple logistic regression analysis in which the presence of mild NAFLD was designated as the dependent variable in three different models (Table 3). FGF21, ALT, AST, GGT, TG, TC, HDL-C, LDL-C, sex, age, and BMI were defined as independent variables in Model 1 . The analysis identified serum FGF21 levels (per $20 \mathrm{pg} / \mathrm{mL}$ increase; odds ratio [OR] 1.082, 95\% confidence interval $[\mathrm{Cl}] 1.020-1.147, P=0.008$ ) as an independent and positive risk factor for NAFLD, along with ALT, 
Table 2. Correlations of IHTC with anthropometric parameters and biochemical indexes in 422 subjects.

\begin{tabular}{|c|c|c|c|c|}
\hline \multirow[t]{2}{*}{ Variables } & \multicolumn{2}{|l|}{ IHTC } & \multicolumn{2}{|c|}{$\begin{array}{l}\text { IHTC (age, BMI } \\
\text { and sex } \\
\text { adjusted) }\end{array}$} \\
\hline & $r$ & $P$ value & $r^{*}$ & $P$ value* \\
\hline $\mathrm{Age}^{\mathrm{a}}$ & -0.009 & 0.86 & & \\
\hline Body mass index & 0.213 & $<0.001$ & & \\
\hline Waist circumference & 0.22 & $<0.001$ & 0.113 & 0.022 \\
\hline Systolic blood pressure ${ }^{a}$ & 0.108 & 0.045 & -0.013 & 0.799 \\
\hline Diastolic blood pressure ${ }^{a}$ & 0.158 & 0.003 & 0.096 & 0.051 \\
\hline Fasting plasma glucose $\mathrm{e}^{\mathrm{a}}$ & -0.014 & 0.802 & 0.021 & 0.669 \\
\hline 2-h plasma glucose ${ }^{a}$ & 0.088 & 0.100 & 0.035 & 0.483 \\
\hline Fasting serum $C$ peptide ${ }^{a}$ & 0.244 & $<0.001$ & 0.359 & $<0.001$ \\
\hline Fasting serum insulin ${ }^{a}$ & 0.107 & 0.063 & 0.086 & 0.103 \\
\hline HOMA-IR ${ }^{\mathrm{a}}$ & 0.087 & 0.129 & 0.078 & 0.142 \\
\hline Alanine transaminase ${ }^{a}$ & 0.356 & $<0.001$ & 0.384 & $<0.001$ \\
\hline Aspartate aminotransferase ${ }^{a}$ & 0.261 & $<0.001$ & 0.282 & $<0.001$ \\
\hline Gamma-glutamyl-transferase ${ }^{a}$ & 0.174 & 0.001 & 0.242 & $<0.001$ \\
\hline Triglyceride $^{a}$ & 0.139 & 0.010 & 0.337 & $<0.001$ \\
\hline Total cholesterol & 0.051 & 0.341 & 0.203 & $<0.001$ \\
\hline $\begin{array}{l}\text { High-density lipoprotein } \\
\text { cholesterol }\end{array}$ & -0.168 & 0.002 & -0.115 & 0.020 \\
\hline $\begin{array}{l}\text { Low-density lipoprotein } \\
\text { cholesterol }\end{array}$ & 0.065 & 0.229 & 0.179 & $<0.001$ \\
\hline Fibroblast growth factor $21^{a}$ & 0.255 & $<0.001$ & 0.275 & $<0.001$ \\
\hline Cytokeratin 18 M65ED & 0.331 & $<0.001$ & 0.256 & $<0.001$ \\
\hline Proteinase $3^{a}$ & 0.068 & 0.209 & 0.008 & 0.871 \\
\hline Neutrophil elastase $^{a}$ & 0.082 & 0.132 & 0.098 & 0.050 \\
\hline alpha-1 antitrypsin ${ }^{a}$ & -0.038 & 0.485 & 0.044 & 0.370 \\
\hline $\mathrm{NE} / \mathrm{A} 1 \mathrm{AT} \mathrm{T}^{\mathrm{a}}$ & 0.080 & 0.107 & 0.061 & 0.226 \\
\hline $\begin{array}{l}\text { IHTC intrahepatic triglyceride } \\
\text { assessment-insulin resistance, } \\
\text { trypsin } \\
{ }^{* P} \text { value: age, body mass index } \\
\text { a Log-transformed before analysi }\end{array}$ & nt, $\mathrm{HO}$ & elastase, & T & $\begin{array}{l}\text { is model } \\
\text { ha- } 1 \text { anti- }\end{array}$ \\
\hline
\end{tabular}

TG, and BMI (all $P<0.05)$. The OR $(95 \% \mathrm{Cl}$ ) for the risk of mild NAFLD per $20 \mathrm{pg} / \mathrm{mL}$ increase of serum FGF21 was 1.082 (1.017-1.151; $P=0.013)$ when CK18 M65ED, diastolic blood pressure, and metabolic syndrome were included in the analysis, in addition to FGF21, ALT, TG, and BMI, in Model 2. To simplify the model, only FGF21, ALT, TG, and BMI, which remained significant in Models 1 and 2, were included in Model 3. Taking the presence of mild NAFLD as the dependent variable (Model 3), the OR $(95 \%$ Cl) for the risk of mild NAFLD per $20 \mathrm{pg} / \mathrm{mL}$ increase of serum FGF21 was $1.062(1.010-1.118 ; P=0.019)$. The model was named the Mild NAFLD Model, which was calculated as follows:

Mild NAFLD Model $=x /(1+x)$,

$x=\mathrm{e}^{(-6.871+0.003 \times \mathrm{FGF} 21+0.079 \times \mathrm{ALT}+1.151 \times \mathrm{TG}+0.163 \times \mathrm{BMI})}$.

Diagnostic effect of FGF21 for mild NAFLD diagnosed by MRS The receiver-operating characteristic curves shown in Fig. 1 represent the predictive accuracy of the Mild NAFLD Model for mild NAFLD diagnosed by MRS. The AUC was $0.853(95 \% \mathrm{Cl}$ $0.816-0.886)$ for the Mild NAFLD Model and $0.637(95 \% \mathrm{Cl}$ $0.589-0.683$ ) for FGF21 alone. The AUC of the Mild NAFLD
Model was larger than that of FGF21 alone $(P<0.0001)$. The AUC of the Mild NAFLD Model was compared with that of the FGF21 Model, which was used to predict ultrasonography-diagnosed NAFLD and was calculated with FGF21 and BMI in a prospective study [15]. In our cohort, the FGF21 Model indicated NAFLD, with an AUC of 0.737 (95\% Cl 0.692-0.779). However, the AUC of the FGF21 Model was significantly smaller than that of the Mild NAFLD Model $(P=0.0001)$. Calculation of the Youden index to find the optimal cut-off value of the Mild NAFLD Model for indicating the occurrence of mild NAFLD produced a value of 0.69 , with a sensitivity of $85.12 \%$ (95\% Cl $81.31 \%-88.92 \%)$, specificity of $70.93 \%$ (95\% Cl $61.33 \%-80.53 \%)$, positive predictive value of $91.96 \%(95 \%$ Cl $88.94 \%-94.98 \%)$, and negative predictive value of $54.95 \%(95 \%$ Cl 45.70\%-64.21\%) (Table 4).

Combined application of ultrasonography and Mild NAFLD Model in diagnosing mild NAFLD

Table 4 summarizes the diagnostic performance of ultrasonography alone, the Mild NAFLD Model alone, and the combination of ultrasonography and the Mild NAFLD Model in diagnosing mild NAFLD using MRS as the reference standard. Ultrasonography or Mild NAFLD Model alone did not show any significant differences regarding the diagnosis of mild NAFLD via the chi-square test. We further evaluated diagnostic performance using a two-step approach in which subjects classified as non-NAFLD by ultrasonography would undergo a secondary assessment by the Mild NAFLD Model using the cutoff value of 0.69. Compared with ultrasonography or the Mild NAFLD Model alone, the two-step approach yielded an overall sensitivity of $97.32 \%(95 \% \mathrm{Cl}$ $95.60 \%-99.05 \%)$ and a negative predictive value of $85.48 \%(95 \%$ Cl 76.72\%-94.25\%), revealing a significant improvement.

\section{DISCUSSION}

In this study, elevated serum FGF21 was the only biomarker independently correlated with IHTC measured by MRS. FGF21 was the only indicator among six potential NAFLD biomarkers that was significantly associated with mild NAFLD diagnosis, and the Mild NAFLD Model based on FGF21 had an AUC of 0.853. Furthermore, the two-step method using the combination of ultrasonography and the Mild NAFLD Model was more sensitive than each method alone to diagnose NAFLD with MRS as the diagnostic reference standard. Elevated FGF21 was independently associated with IHTC in mild NAFLD and was also increased in NAFLD before ultrasonography was performed. Increased hepatic TG deposition reflects an imbalance of hepatic free fatty acid (FFA) metabolism [24]. FFAs are natural agonists of peroxisome proliferator-activated receptor-a (PPARa), and hepatic Fgf21 expression is upregulated by the transcription factor PPARa [25]. In addition, a study showed that hepatic FGF21 was increased during nutrient overload and was involved in de novo lipogenesis in the liver in response to carbohydrate load [25].

Although CK18 M65ED was elevated in mild NAFLD in our study, it was not independently associated with IHTC. CK18 M65ED, a CK18 fragment, can detect the common epitope in both uncleaved and caspase-cleaved CK18, reflects hepatocellular apoptosis and is potentially useful for diagnosing NAFLD $[26,27]$. Unlike FGF21, the elevated CK18 M65ED in our study might reflect the increasing level of apoptosis-induced total cell death, which might be the result of cellular stress and hepatic injury subsequently induced by the hepatic accumulation of lipids [28]. This may explain why CK18 M65ED increased but was not correlated with IHTC in mild NAFLD in our population.

An animal study revealed that the activation of PR3 can contribute to NAFLD and insulin resistance, and PR3-deficient mice showed reduced intrahepatic lipids and were resistant to high-fat-diet-induced weight gain [18]. NE can cause cellular insulin resistance, and the deletion of NE in mice can ameliorate 
Table 3. Multivariate logistic regression for the association between serum FGF21 levels and mild NAFLD in 422 subjects.

\begin{tabular}{|c|c|c|c|c|}
\hline Variables & $\beta$ & Standard error & Odds ratios $(95 \% \mathrm{Cl})$ & $P$ value \\
\hline \multicolumn{5}{|l|}{ Model 1} \\
\hline Fibroblast growth factor $21^{\mathrm{a}}(\mathrm{pg} / \mathrm{mL})$ & 0.079 & 0.030 & $1.082(1.020-1.147)$ & 0.008 \\
\hline Triglycerides (mmol/L) & 0.950 & 0.340 & $2.587(1.328-5.040)$ & 0.005 \\
\hline Body mass index $\left(\mathrm{kg} / \mathrm{m}^{2}\right)$ & 0.139 & 0.056 & $1.149(1.029-1.283)$ & 0.013 \\
\hline Alanine transaminase $(\mathrm{U} / \mathrm{L})$ & 0.119 & 0.032 & $1.127(1.058-1.199)$ & $<0.001$ \\
\hline Triglycerides (mmol/L) & 0.974 & 0.364 & $2.649(1.298-5.403)$ & 0.007 \\
\hline Body mass index $\left(\mathrm{kg} / \mathrm{m}^{2}\right)$ & 0.145 & 0.061 & $1.156(1.026-1.303)$ & 0.018 \\
\hline \multicolumn{5}{|l|}{ Model 3} \\
\hline Fibroblast growth factor $21^{\mathrm{a}}(\mathrm{pg} / \mathrm{mL})$ & 0.061 & 0.026 & $1.062(1.010-1.118)$ & 0.019 \\
\hline \multicolumn{5}{|c|}{$\begin{array}{l}\text { Original variables included: } \\
\text { Model 1: FGF21, ALT, AST, GGT, TG, TC, HDL-C, LDL-C, adjusted by sex, age, and BMI } \\
\text { Model 2: FGF21, ALT, AST, GGT, TG, TC, HDL-C, LDL-C, DBP, CK18 M65ED, adjusted by sex, age, BMl, and metabolic syndrome } \\
\text { Model 3: FGF21, ALT, TG, and BMI } \\
\text { FGF21 fibroblast growth factor 21, ALT alanine transaminase, AST aspartate aminotransferase, GGT gamma-glutamyl-transferase, TG triglyceride, TC total cholesterol, } \\
\text { HDL-C high-density lipoprotein cholesterol, LDL-C low-density lipoprotein cholesterol, DBP diastolic blood pressure, CK18 cytokeratin } 18, B M I \text { body mass index } \\
\text { aPer } 20 \mathrm{pg} / \mathrm{mL}\end{array}$} \\
\hline
\end{tabular}

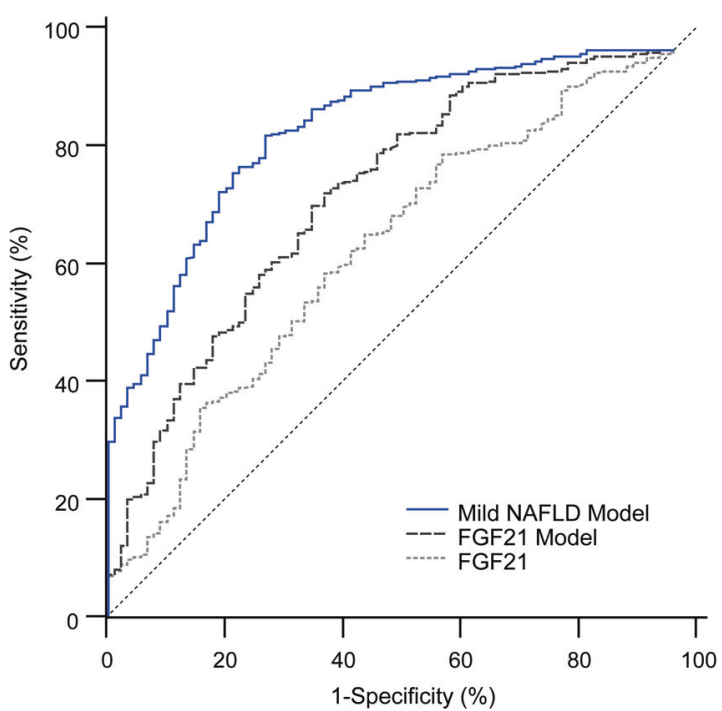

Fig. 1 ROC curves for predicting MRS-diagnosed mild NAFLD and the comparison of AUCs. Mild NAFLD Model (FGF21 + ALT + TG + $\mathrm{BMI}), \mathrm{AUC}=0.853(95 \% \mathrm{Cl}$ 0.816-0.886); FGF21 Model (FGF21 + $\mathrm{BMI}), \mathrm{AUC}=0.737(95 \% \mathrm{Cl} 0.692-0.779) ; \mathrm{FGF} 21, \mathrm{AUC}=0.637(95 \%$ Cl 0.589-0.683). Comparison of AUCs: Mild NAFLD Model vs. FGF21 Model, $P<0.0001$; Mild NAFLD Model vs. FGF21, $P<0.0001$; FGF21 Model vs. FGF21, $P=0.0042$.

tissue inflammation [19]. A1AT is an endogenous inhibitor of NE, and the imbalance between NE and A1AT is associated with obesity-related metabolic inflammation [20]. Zang et al. found an elevation in serum NE and NE/A1AT and a reduction of A1AT in NASH subjects [21]. However, in our current study, PR3, NE, A1AT, and NE/A1AT were not significantly altered in patients with mild NAFLD. This might be explained by the 'multiple hit hypothesis' [29] where, in mild NAFLD, the first hit of rising hepatic lipid concentrations had rendered hepatocytes sensitive to an additional hit featuring hepatocyte cell death, but it had not yet driven drastic changes in hepatic inflammation.

In our study, the two-step method, which involved combining serum biomarkers with conventional ultrasonography, resulted in good sensitivity in mild NAFLD. In this study, the two-step method achieved a sensitivity of $97.32 \%$ and a negative predictive value of $85.48 \%$ for the diagnosis of mild NAFLD. Ultrasonography alone has well-known limitations in detecting IHTC, with sensitivities of $65.1 \%$ and $64.9 \%$ in the assessment of mild NAFLD patients [30] and severely obese patients [31], respectively. However, ultrasonography has some advantages. It is not influenced by liver iron [30], it is easily accessible and inexpensive, and it is particularly useful for the general screening of subjects compared with other imaging techniques (i.e., CT or MRS) [11]. In addition, ultrasonography can be performed easily in conscious patients, which is an advantage when examining pediatric patients or adults unable to tolerate magnetic resonance imaging tests without sedation [32].

The Mild NAFLD Model, which is the other component of the two-step method and was obtained using MRS as a reference standard, is composed of FGF21, ALT, TG, and BMI, which are all easily obtained indices in clinical practice. Other biomarkers, such as the fatty liver index and the hepatic steatosis index, also have moderate accuracy in diagnosing NAFLD [33-35]. However, the key limitation of these two indices is their use of ultrasonography, which is a suboptimal reference standard [35]. Hopefully, by adopting the two-step method in diagnosing mild NAFLD, suggestions on lifestyle interventions regarding diet and physical activity can be provided to these subjects to prevent the deterioration of NAFLD.

This study has several limitations. First, longitudinal data were lacking; thus, we could not address the causal relationship between FGF21 and mild NAFLD. Prospective studies in larger populations are needed to confirm our diagnostic method. Second, a liver biopsy was not used, and information concerning coexisting steatohepatitis or fibrosis was lacking. Third, the diagnostic accuracy of NAFLD via MRS could have been affected 
Diagnosis of NAFLD by combination methods

LL Qian et al.

Table 4. Improved performance in diagnosing mild NAFLD by the two-step approach by combining ultrasonography and Mild NAFLD Model.

\begin{tabular}{|c|c|c|c|c|c|c|}
\hline Variables & $\begin{array}{l}\text { Ultrasonography } \\
(95 \% \mathrm{Cl})\end{array}$ & $\begin{array}{l}\text { Mild NAFLD Model } \\
(95 \% \mathrm{Cl})\end{array}$ & $\begin{array}{l}\text { Two-step approach } \\
(95 \% \mathrm{Cl})\end{array}$ & $P$ value* & $P$ value ${ }^{* *}$ & $P$ value $* * *$ \\
\hline True positive & 303 & 286 & 327 & - & - & - \\
\hline True negative & 56 & 61 & 53 & - & - & - \\
\hline False negative & 33 & 50 & 9 & - & - & - \\
\hline Sensitivity (\%) & $90.18(87.00-93.36)$ & $85.12(81.31-88.92)$ & $97.32(95.60-99.05)$ & 0.264 & 0.045 & 0.003 \\
\hline Positive predictive value (\%) & 90.99 (87.92-94.07) & $91.96(88.94-94.98)$ & $90.83(87.85-93.81)$ & 0.800 & 0.809 & 1.000 \\
\hline Negative predictive value (\%) & $62.92(52.89-72.96)$ & $54.95(45.70-64.21)$ & $85.48(76.72-94.25)$ & 0.250 & $<0.001$ & $<0.001$ \\
\hline
\end{tabular}

by hepatic iron content [36]. However, it was not possible to exclude the influence of hepatic iron because of insufficient data. In addition, one major limitation of the study is that the diagnostic performance of the Mild NAFLD Model had not been validated in another external validation population. In addition, our study population may not represent the general Chinese community population. Therefore, the conclusion needs further validation in a general population. Last, the MRS diagnosis was based on data collected from a small portion of the liver (within a voxel $=2 \mathrm{~cm} \times$ $2 \mathrm{~cm} \times 2 \mathrm{~cm}$ ), and this might have led to a sampling error [35].

In conclusion, the present study demonstrated that elevated serum FGF21 was independently associated with IHTC as measured by MRS and was indicative of mild NAFLD compared with the other five potential NAFLD biomarkers. A two-step approach that combined ultrasonography and the Mild NAFLD Model derived from serum FGF21 further improved the diagnostic sensitivity for mild NAFLD. We believe this diagnostic model can be applied in the early diagnosis of NAFLD in clinical practice.

\section{ACKNOWLEDGEMENTS}

This work was supported by the National Natural Science Foundation Major International (Regional) Joint Research Project (81220108006) and an NSFC-NHMRC Joint Research grant (81561128016) to WPJ; the General Program of Natural Science Foundation of China (81870598), the Shanghai Pujiang Program (17PJ1407500), and the Municipal Human Resources Development Program for Outstanding Young Talents in Medical Health Sciences in Shanghai (2017YQ009) to HTL; and the Shanghai Municipal Key Clinical Specialty.

\section{AUTHOR CONTRIBUTION}

LLQ and LW performed the data analysis and wrote the manuscript; LLQ, LZ, and JZ contributed to the acquisition of data; LW assisted in statistical analysis; QCF and HTL contributed to data interpretation; JZ and YHL assisted the magnetic resonance spectroscopy examinations; HTL and WPJ were in charge of the overall conception and design of the study. All authors included in the authorship list approved the final manuscript version.

\section{ADDITIONAL INFORMATION}

Competing interests: The authors declare no competing interests.

\section{REFERENCES}

1. Younossi Z, Tacke F, Arrese M, Sharma BC, Mostafa I, Bugianesi E, et al. Global perspectives on non-alcoholic fatty liver disease and non-alcoholic steatohepatitis. Hepatology. 2018;69:2672-82.

2. Kleiner DE, Brunt EM, Van Natta M, Behling C, Contos MJ, Cummings OW, et al. Design and validation of a histological scoring system for nonalcoholic fatty liver disease. Hepatology. 2005;41:1313-21.
3. Adams LA, Sanderson S, Lindor KD, Angulo P. The histological course of nonalcoholic fatty liver disease: a longitudinal study of 103 patients with sequential liver biopsies. J Hepatol. 2005;42:132-8.

4. Chalasani N, Wilson L, Kleiner DE, Cummings OW, Brunt EM, Unalp A. Relationship of steatosis grade and zonal location to histological features of steatohepatitis in adult patients with non-alcoholic fatty liver disease. J Hepatol. 2008;48:829-34.

5. McPherson S, Hardy T, Henderson E, Burt AD, Day CP, Anstee QM. Evidence of NAFLD progression from steatosis to fibrosing-steatohepatitis using paired biopsies: implications for prognosis and clinical management. J Hepatol. 2015;62:1148-55.

6. Wong VW, Wong GL, Choi PC, Chan AW, Li MK, Chan HY, et al. Disease progression of non-alcoholic fatty liver disease: a prospective study with paired liver biopsies at 3 years. Gut. 2010;59:969-74.

7. Anstee QM, Targher G, Day CP. Progression of NAFLD to diabetes mellitus, cardiovascular disease or cirrhosis. Nat Rev Gastroenterol Hepatol. 2013;10:330-44.

8. Shen X, Cai J, Gao J, Vaidya A, Liu X, Li W, et al. Nonalcoholic fatty liver disease and risk of diabetes: a prospective study in China. Endocr Pract. 2018;24:823-32.

9. Bohte $A E$, van Werven JR, Bipat $S$, Stoker J. The diagnostic accuracy of US, CT, MRI and 1H-MRS for the evaluation of hepatic steatosis compared with liver biopsy: a meta-analysis. Eur Radiol. 2011;21:87-97.

10. Takyar V, Nath A, Beri A, Gharib AM, Rotman Y. How healthy are the "Healthy volunteers"? Penetrance of NAFLD in the biomedical research volunteer pool Hepatology. 2017;66:825-33.

11. Hernaez R, Lazo M, Bonekamp S, Kamel I, Brancati FL, Guallar E, et al. Diagnostic accuracy and reliability of ultrasonography for the detection of fatty liver: a metaanalysis. Hepatology. 2011;54:1082-90.

12. Lee SS, Park SH, Kim HJ, Kim SY, Kim MY, Kim DY, et al. Non-invasive assessment of hepatic steatosis: prospective comparison of the accuracy of imaging examinations. J Hepatol. 2010;52:579-85.

13. Machado MV, Cortez-Pinto H. Non-invasive diagnosis of non-alcoholic fatty liver disease. A critical appraisal. J Hepatol. 2013;58:1007-19.

14. Hetterich $H$, Bayerl C, Peters A, Heier M, Linkohr B, Meisinger C, et al. Feasibility of a three-step magnetic resonance imaging approach for the assessment of hepatic steatosis in an asymptomatic study population. Eur Radiol. 2016;26:1895-904.

15. Woo YC, Xu A, Wang Y, Lam KS. Fibroblast growth factor 21 as an emerging metabolic regulator: clinical perspectives. Clin Endocrinol. 2013;78:489-96.

16. Li H, Dong K, Fang Q, Hou X, Zhou M, Bao Y, et al. High serum level of fibroblast growth factor 21 is an independent predictor of non-alcoholic fatty liver disease: a 3-year prospective study in China. J Hepatol. 2013;58:557-63.

17. Yan $H, X i a ~ M, C h a n g ~ X, X u Q$, Bian $H$, Zeng $M$, et al. Circulating fibroblast growth factor 21 levels are closely associated with hepatic fat content: a cross-sectional study. PLoS One. 2011;6:e24895.

18. Toonen EJ, Mirea AM, Tack CJ, Stienstra R, Ballak DB, van Diepen JA, et al. Activation of proteinase 3 contributes to non-alcoholic fatty liver disease (NAFLD) and insulin resistance. Mol Med. 2016;22:202-14.

19. Talukdar S, Oh DY, Bandyopadhyay G, Li D, Xu J, McNelis J, et al. Neutrophils mediate insulin resistance in mice fed a high-fat diet through secreted elastase. Nat Med. 2012;18:1407-12.

20. Mansuy-Aubert V, Zhou OL, Xie X, Gong Z, Huang JY, Khan AR, et al. Imbalance between neutrophil elastase and its inhibitor alpha1-antitrypsin in obesity alters insulin sensitivity, inflammation, and energy expenditure. Cell Metab. 2013;17:534-48.

21. Zang S, Ma X, Zhuang Z, Liu J, Bian D, Xun Y, et al. Increased ratio of neutrophil elastase to alpha1-antitrypsin is closely associated with liver inflammation in 
patients with nonalcoholic steatohepatitis. Clin Exp Pharmacol Physiol. 2016;43:13-21.

22. Alkhouri N, Carter-Kent C, Feldstein AE. Apoptosis in nonalcoholic fatty liver disease: diagnostic and therapeutic implications. Expert Rev Gastroenterol Hepatol. 2011;5:201-12.

23. Szczepaniak LS, Nurenberg P, Leonard D, Browning JD, Reingold JS, Grundy S, et al. Magnetic resonance spectroscopy to measure hepatic triglyceride content: prevalence of hepatic steatosis in the general population. Am J Physiol Endocrinol Metab. 2005;288:E462-8.

24. Rotman Y, Sanyal AJ. Current and upcoming pharmacotherapy for non-alcoholic fatty liver disease. Gut. 2017;66:180-90.

25. Fisher FM, Maratos-Flier E. Understanding the physiology of FGF21. Annu Rev Physiol. 2016;78:223-41.

26. Joka D, Wahl K, Moeller S, Schlue J, Vaske B, Bahr MJ, et al. Prospective biopsycontrolled evaluation of cell death biomarkers for prediction of liver fibrosis and nonalcoholic steatohepatitis. Hepatology. 2012;55:455-64.

27. Wu G, Li H, Fang Q, Zhang J, Zhang M, Zhang L, et al. Complementary role of fibroblast growth factor 21 and cytokeratin 18 in monitoring the different stages of nonalcoholic fatty liver disease. Sci Rep. 2017;7:5095.

28. Lebeaupin C, Vallee D, Hazari Y, Hetz C, Chevet E, Bailly-Maitre B. Endoplasmic reticulum stress signalling and the pathogenesis of non-alcoholic fatty liver disease. J Hepatol. 2018;69:927-47.

29. Buzzetti E, Pinzani M, Tsochatzis EA. The multiple-hit pathogenesis of nonalcoholic fatty liver disease (NAFLD). Metabolism. 2016;65:1038-48.
30. Kromrey ML, Ittermann $T$, Berning $M$, Kolb C, Hoffmann RT, Lerch MM, et al. Accuracy of ultrasonography in the assessment of liver fat compared with MRI. Clin Radiol. 2019;74:539-46.

31. de Moura Almeida A, Cotrim HP, Barbosa DB, de Athayde LG, Santos AS, Bitencourt AG, et al. Fatty liver disease in severe obese patients: diagnostic value of abdominal ultrasound. World J Gastroenterol. 2008;14:1415-8.

32. Ozturk A, Grajo JR, Gee MS, Benjamin A, Zubajlo RE, Thomenius KE, et al. Quantitative hepatic fat quantification in non-alcoholic fatty liver disease using ultrasound-based techniques: a review of literature and their diagnostic performance. Ultrasound Med Biol. 2018;44:2461-75.

33. Bedogni G, Bellentani S, Miglioli L, Masutti F, Passalacqua M, Castiglione A, et al. The Fatty Liver Index: a simple and accurate predictor of hepatic steatosis in the general population. BMC Gastroenterol. 2006;6:33.

34. Lee JH, Kim D, Kim HJ, Lee CH, Yang Jl, Kim W, et al. Hepatic steatosis index: a simple screening tool reflecting nonalcoholic fatty liver disease. Dig Liver Dis. 2010;42:503-8.

35. Wong VW, Adams LA, de Ledinghen V, Wong GL. Noninvasive biomarkers in NAFLD and NASH-current progress and future promise. Nat Rev Gastroenterol Hepatol. 2018;15:461-78.

36. McPherson S, Jonsson JR, Cowin GJ, O'Rourke P, Clouston AD, Volp A, et al. Magnetic resonance imaging and spectroscopy accurately estimate the severity of steatosis provided the stage of fibrosis is considered. J Hepatol. 2009;51: 389-97. 